\title{
Palatal Swab or Saliva: A Researcher's Quest for Candida Count in Healthy Edentulous Individuals
}

\author{
${ }^{1}$ Tamanna Chhabra, ${ }^{2}$ Vandana Shah, ${ }^{3}$ JR Patel
}

\begin{abstract}
Aim: Many techniques have been used for quantification of Candida colony-forming unit (CFU) in the oral cavity. Also it is proved that unstimulated saliva and concentrated oral rinse show maximum fungal loading. Healthy edentulous patients prior to wearing complete denture do not possess a Candida lesion or accretion of Candida colonies in the palate. Therefore, an appropriate technique to find out the CFU of Candida in such cases is still not clear. Hence, the aim of this study was to determine if palatal swab buffered in saline can be one of the most appropriate techniques to find out CFU of Candida in healthy edentulous patients.
\end{abstract}

Materials and methods: This was done by comparing CFU count obtained in unstimulated saliva and a palatal swab buffered in phosphate saline in 40 healthy edentulous patients approaching the outpatient department (OPD). Both the samples collected were inoculated on Sabouraud dextrose agar (SDA) plates for 24 hours. The CFU was counted in digital colony counter.

Results: Paired t-test shows that there is statistically no difference in CFU count obtained from both the methods. The Pearson's correlation coefficient shows a statistically significant positive correlation $(p<0.05)$ between the two types of sampling techniques.

Conclusion: From the results it can be concluded that palatal swab buffered in saline can be used as one of the techniques to quantify Candida than plain palatal swab which though site specific is not a quantifying technique for the same.

Clinical significance: Using palatal swab buffered in saline technique will help the clinicians to obtain the samples from healthy edentulous patients in the studies involving quantification of Candida.

Keywords: Candida, Colony-forming unit, Edentulous, Palatal swab, Saliva.

How to cite this article: Chhabra T, Shah V, Patel JR. Palatal Swab or Saliva: A Researcher's Quest for Candida Count in Healthy Edentulous Individuals. World J Dent 2018;9(2):87-90.

\footnotetext{
${ }^{1}$ Department of Prosthodontics, K M Shah Dental College \& Hospital, Sumandeep Vidyapeeth, Vadodara, Gujarat, India

${ }^{2}$ Department of Oral Pathology, K M Shah Dental College \& Hospital, Sumandeep Vidyapeeth, Vadodara, Gujarat, India

${ }^{3}$ Department of Prosthodontics, Narsinhbhai Patel Dental College \& Hospital, Sankalchand Patel University, Visnagar Gujarat, India

Corresponding Author: Tamanna Chhabra, Department of Prosthodontics, K M Shah Dental College \& Hospital Sumandeep Vidyapeeth, Vadodara, Gujarat, India, e-mail: drtamannachhabra@gmail.com
}

Source of support: Nil

Conflict of interest: None

\section{INTRODUCTION}

Saliva plays an important role in the oral cavity of an individual. Among its other constituents, microorganisms normally exist as commensals in the oral cavity. Candida are one group of organisms that constitute the saliva and are opportunistic organisms. When the defense mechanism of the body lowers due to any cause, the Candida increase the risk of infections. Also with aging the functioning of the body's immune system slows down and can put an individual to the risk of infections. This along with the edentulous state of an elderly person predisposes to flared opportunistic behavior of Candida. Reports from studies show that persons wearing dentures have increased complex flora than their healthy counterparts. ${ }^{1,2}$ Moreover, prolonged wearing of prosthesis also poses a major risk factor for colonization of Candida on the oral mucosal surfaces ${ }^{3}$ and may lead to denture stomatitis, resulting in inflamed and erythematous palate. It may also be associated with angular cheilitis.

In order for a clinician to decide about the virulence of Candida in edentulous patients, quantitative estimation of the fungal load could help in identifying the risk of fungal infections associated with it. ${ }^{4}$ This quantification needs to be done to differentiate between Candida carriage and its pathogenicity and can be done using any of these techniques like imprints, concentrated oral rinses, unstimulated saliva, swab, and culture.

Many a times, studies are planned wherein both the types of techniques are used to find out the colonization of Candida, which can be time- and resource-consuming. As in a study done by Borole et $\mathrm{al}^{5}{ }^{5}$ swab and unstimulated saliva samples were taken in diabetic and nondiabetic edentulous patients to estimate the CFU of Candida. In the past, there have been studies which proved that there is a strong correlation between all the techniques of quantitatively measuring $\mathrm{CFU} / \mathrm{mL}$ of Candida from the oral cavity. Tooyama et $\mathrm{al}^{6}$ did a study to find out the most relevant method of quantifying $\mathrm{CFU} / \mathrm{mL}$ on patients approaching the dental OPD. The study showed that concentrated oral rinse gives the maximum amount of CFU/mL of Candida. Therefore, quantification of Candida by taking a swab and inoculating it does not 
assure the quantity of Candida intraorally. But if the swab is buffered in saline, then it becomes a quantifiable liquid and can be compared with the same amount of oral rinse and unstimulated saliva. There are no studies where the palatal swab buffered with saline is compared for CFU/mL of Candida with unstimulated saliva. Also, there are no studies done on healthy edentulous patients who are Candida carriers and not suffering from any chronic illness. Healthy edentulous patients may not have a lesion or accretion of Candida colonies in the palate. Appropriate sampling technique used to collect data can be utilized as baseline for further comparative researches involving quantification of Candida. Therefore, the aim was to determine if palatal swab buffered in saline can be one of the most appropriate techniques in healthy edentulous patients which is site specific as well as gives the exact CFU/mL of Candida in the oral cavity of healthy edentulous patients. This was done by checking if there is any relation between the quantity of Candida CFU/mL count in unstimulated saliva and a palatal swab buffered in phosphate saline in a healthy edentulous patient.

\section{MATERIALS AND METHODS}

A total of 40 completely edentulous patients attending the OPD of the Department of Prosthodontics were enrolled in the study. After explaining the significance and purpose of the study, a written informed consent was obtained from each participant. A sterile cotton swab was used to obtain the sample from the palatal mucosa, by scrapping for 10 seconds. The sample was diluted in phosphate buffered saline and $20 \mu \mathrm{L}$ was inoculated on SDA plates with 100 mg chloramphenicol/L (brand). After incubation for 24 to 48 hours, colonies were observed and counted using a colony counter, determining the number of CFU/mL. ${ }^{7}$

For the second method, participants were asked to rinse their oral cavity with water, and $2 \mathrm{~mL}$ of unstimulated whole saliva was allowed to drool in a disposable sterilize plastic container; $20 \mu \mathrm{L}$ was pipetted out of the container and inoculated on the SDA plates with $100 \mathrm{mg}$ chloramphenicol/L. After incubation for 24 to 48 hours, colonies were counted, and the number of $\mathrm{CFU} / \mathrm{mL}$ was determined. 8,9

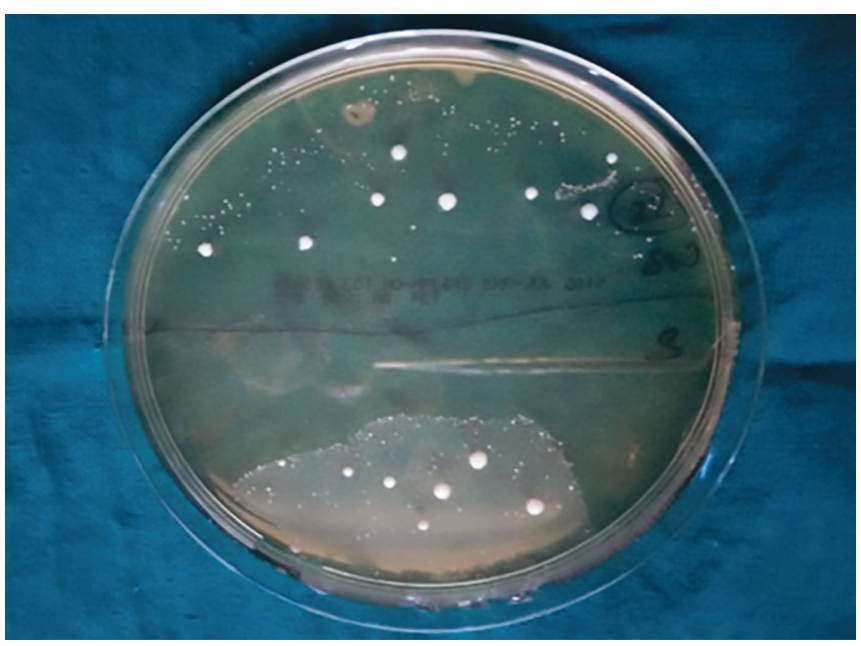

Fig. 1: Colony-forming units in swab and saliva

Table 1: Mean and standard deviation for CFU in swab and CFU in saliva

\begin{tabular}{lllll}
\hline Variables & Mean & $n$ & Std. deviation & Std. error mean \\
\hline CFU in swab & 73.1000 & 40 & 58.89269 & 9.31175 \\
CFU in saliva & 58.8500 & 40 & 39.52185 & 6.24895 \\
\hline
\end{tabular}

Statistical analysis was done using Pearson correlation coefficient to find out the relation between the two different quantification techniques used for counting $\mathrm{CFU} / \mathrm{mL}$ of Candida. Also paired t-test was used on the data obtained from the patients.

\section{RESULTS}

The study compared the relation between CFU/mL of Candida in palatal swab buffered in phosphate saline and unstimulated saliva in healthy edentulous patients. Figure 1 shows the comparison between CFU/mL in unstimulated saliva and palatal swab on either half of the plates. Mean and standard deviation for CFU/mL in swab is 73.00 and mean CFU/mL in saliva is 58.8 (Table 1 ).

Paired t-test is shown in Table 2, which shows that the mean CFU/mL in swab is more than in saliva with a mean difference of $14.25 \pm 50.63$ and the p-value is also $>0.05$, showing that there is no statistically significant difference between the CFU/mL counts in swab and saliva.

The Pearson's correlation coefficient was applied to the data recorded from both the types of sampling techniques.

Table 2: Paired t-test for CFU in swab and CFU in saliva

\begin{tabular}{|c|c|c|c|c|c|c|c|c|c|}
\hline \multicolumn{10}{|c|}{ Paired samples test } \\
\hline & & \multicolumn{5}{|c|}{ Paired differences } & \multirow[b]{3}{*}{$t$-value } & \multirow[b]{3}{*}{$d f$} & \multirow[b]{3}{*}{$p$-value } \\
\hline & & \multirow[b]{2}{*}{ Mean } & \multirow[b]{2}{*}{ Std. deviation } & \multirow[b]{2}{*}{ Std. error mean } & \multicolumn{2}{|c|}{$\begin{array}{c}95 \% \text { confidence interval } \\
\text { of the difference }\end{array}$} & & & \\
\hline & & & & & Lower & Upper & & & \\
\hline Pair 1 & $\begin{array}{l}\text { CFU in swab - CFU in } \\
\text { saliva }\end{array}$ & 14.25000 & 50.63887 & 8.00671 & -1.94510 & 30.44510 & 1.780 & 39 & 0.083 \\
\hline
\end{tabular}




\begin{tabular}{llll}
\hline \multicolumn{2}{c}{ Table 3: Correlation between CFU in palatal swab and saliva } \\
\hline & & \multicolumn{2}{c}{ CFU in } \\
swab & $\begin{array}{l}\text { CFU in } \\
\text { saliva }\end{array}$ \\
\hline CFU in swab & Pearson correlation & 1 & $0.530^{*}$ \\
& Sig. (2-tailed) & & 0.000 \\
& $\mathrm{n}$ & 40 & 40 \\
CFU in saliva & Pearson correlation & $0.530^{*}$ & 1 \\
& Sig. (2-tailed) & 0.000 & \\
& $\mathrm{n}$ & 40 & 40 \\
\hline
\end{tabular}

${ }^{*}$ Correlation is significant at the 0.01 level (2-tailed)

Table 3 shows the mean and standard deviation for the groups of samples of $\mathrm{CFU} / \mathrm{mL}$ counted in palatal swab and unstimulated saliva. The result of the study shows a statistically significant positive correlation $(\mathrm{p}<0.05)$ between the two types of sampling techniques. The Pearson correlation is 0.530 , which is in the category of moderate correlation and this is significant as p-value is 0.000 .

\section{DISCUSSION}

Evaluation of amount of Candida in edentulous patients is one of the commonest techniques used in several studies done on edentulous patients. There are several sample collecting techniques described and used variedly by different researchers. Various techniques used for counting CFUs of Candida are by taking palatal swab, imprint culture, unstimulated saliva, and concentrated oral rinse. Each technique has its advantages and disadvantages and there is no clear information as to which is the superior technique to count the CFU of Candida intraorally, especially in a healthy edentulous patient since most of the studies are carried out on patients suffering from oral candidiasis. ${ }^{10}$

A study conducted in 2015 has shown that maximum fungal loading is in concentrated oral rinse. ${ }^{11,12}$ But in an edentulous patients, there is a need to be localized to palatal mucosa as the palatal swab collected can be compared with the swab collected in future after wearing the denture. It may serve as the baseline for further research on denture stomatitis which is seen after the development of the disease. Therefore, this study was planned to assess whether the palatal swab buffered in saline can be used as a technique for quantification of Candida in healthy edentulous patients. A study also shows Candida has capacity to adhere to oral epithelial cells and denture which is in contact with denture surface. ${ }^{13,14}$

Concentrated oral rinse is considered superior for quantification but involves centrifuging the oral rinse. ${ }^{10}$ Unstimulated saliva is equally sensitive but not site-specific. Swab is a relatively simple but may not be a standardized technique and is usually used to just confirm the presence of Candida and is not a complete quantification technique. Therefore palatal swab alone is not recommended as standard technique for quantifying CFU/mL of Candida.
Many studies have used either one of the techniques to count the fungal loading, which may not be the most appropriate for the patients on whom the $\mathrm{CFU} / \mathrm{mL}$ of Candida is being obtained. Few studies have used both techniques simultaneously which is time- and resourceconsuming. Therefore, this study was planned to ascertain the appropriate technique for quantifying $\mathrm{CFU} / \mathrm{mL}$ of Candida in healthy edentulous patients by relating the two sampling techniques. In order to standardize both the techniques as liquid quantification, palatal swab was buffered in $20 \mu \mathrm{L}$ of phosphate saline once. Then the similar quantity of saline as well as unstimulated saliva was inoculated on SDA for 24 to 48 hours.

The results of this study show a positive relation between both the techniques, although the relation is moderate. This may be due to converting a simple swab to a measurable liquid by buffering in phosphate saline. The result shows a statistically significant positive correlation $(\mathrm{p}<0.05)$ between the two types of sampling techniques.

The result of this study is in accordance with the other studies. A study conducted by Kimori et $\mathrm{al}^{11}$ showed that swab and unstimulated saliva give similar amount of Candida but less when compared with concentrated oral rinse. Alazzawi et $\mathrm{al}^{8}$ also proved the same in their study.

The study was carried out on healthy edentulous patients. Healthy edentulous patients were chosen in order to establish the baseline data with appropriate technique before any interference of dentures, and change in microflora is seen in the oral cavity. Then the study can be carried out further with either technique after the wearing of the dentures.

Paired $\mathrm{t}$-test shows that there is no statistically significant difference between the two sampling techniques ( $p>0.05)$. Therefore, either of the techniques can be used interchangeably. Though palatal swab showed more amount of $\mathrm{CFU} / \mathrm{mL}$ than unstimulated saliva, it could be due to adherence of Candida to the palate.

Studies have utilized this technique of buffering swab in phosphate saline, but no comparison between the data in healthy edentulous patients has been made. Physical impaction of swab also destroys the viable Candida count and disrupts the agar plate. ${ }^{15}$ Buffering the swab sample in saline makes the entry of all the cells into liquid smooth and it becomes easy to count the number of cells in such a sample. Direct inoculation of swab onto the SDA plate will retain a few cells in the swab. Also quantification of cells in certain amount of liquid is comparatively accurate, it also is nontoxic to the viable cells.

Limitation of the study is that this was the only relative study of comparison between the two techniques. Further studies can be planned where palatal swab buffered in saline can be compared with concentrated oral 
rinse technique, which gives even more Candida counts with even larger sample size.

\section{CONCLUSION}

The study concludes that for the quantification of CFUs of Candida in a healthy edentulous patients, the palatal swab buffered in saline technique can be used in comparison to dry palatal swab or unspecific samples of unstimulated saliva and concentrated oral rinse.

\section{CLINICAL SIGNIFICANCE}

The results of this study reveal that the clinicians and researchers can utilize the palatal swab buffered in saline sampling method in healthy edentulous patients for counting CFU/mL of Candida for diagnosis or research purposes.

\section{REFERENCES}

1. Budtz-Jorgensen E, Stenderup A, Grabowski M. An epidemiology study of yeasts in elderly denture wearers. Community Dent Oral Epidemiol 1975 May;3(3):115-119.

2. Beighton D, Ludford R, Clark DT, Brailsford SR, Pankhurst CL, Tinsley GF, Fiske J, Lewis D, Daly B, Khalifa N, et al. Use of CHROM agar Candida medium for isolation of yeast from dental samples. J Clin Microbiol 1995 Nov;33(11):3025-3027.

3. Arendorf TM, Addy M. Candidal carriage and plaque distribution before, during, and after removable orthodontic appliance therapy. J Clin Periodontol 1985 May;12(5): 360-368.

4. Muneer MU, Qamar K, Naeem S, Haroon S. Candidal count in patients with complete dental prostheses. Pak Oral Dent J 2011 Jun;31(1):207-209.

5. Borole A, Roopa KT, Khandelwal PV. A comparative evaluation of the effects of different commercially available denture adhesives on the growth of Candida species in diabetic and nondiabetic subjects: an in vivo study. J Dent Allied Sci 2017 Sep;5(2):63-69.

6. Tooyama H, Matsumoto T, Hayashi K, Kurashina K, Kurita H, Uchida M, Kasuga E, Honda T. Candida concentrations determined following concentrated oral rinse culture reflect clinical oral signs. BMC Oral Health 2015 Nov;15:150.

7. Ishikawa KH, Mayer MP, Miyazima TY, Matsubara VH, Silva EG, Paula CR, Campos TT, Nakamae AE. A multispecies probiotic reduces oral Candida colonization in denture wearers. J Prosthodont 2015 Apr;24(3):194-199.

8. Alazzawi H, Ahmed JN. Assessment of oral sample collection technique for the isolation of Candida albicans from patients having denture stomatitis. Int J Sci Res 2017 Apr;6(4): 1100-1103.

9. Mahmoudabadi AZ, Drucker DB, Mandall N, O'Brien K, Theaker E. Isolation and identification of Candida species from the oral cavity using CHROM agar Candida. Iran Biomed J 2000 Jul;4(2-3):57-61.

10. Byadarahally Raju S, Rajappa S. Isolation and identification of Candida from the oral cavity. ISRN Dent 2011 Oct;2011:487921.

11. Kimori H, Nakagawa Y, Yamamoto K. Establishing the cut off point for the Candida swab test for daily oral care in dry mouth patients. Oral Ther Pharmacol 2009 Dec;28(1):17-25.

12. Marsh P, Martin M. Oral fungal infections. In: Oral microbiology. Edinburgh: Churchill Livingstone; 2009. p. 166-179.

13. Webb BC, Thomas CJ, Willcox MD, Harty DW, Knox KW. Candida-associated denture stomatitis. Aetiology and management: a review. Part 1. Factors influencing distribution of Candida species in the oral cavity. Aust Dent J 1998 Feb;43(1):45-50.

14. King RD, Lee JC, Morris AL. Adherence of Candida albicans and other Candida species to mucosal epithelial cells. Infect Immun $1980 \mathrm{Feb}$;27(2):667-674.

15. Thomas P, Mujawar MM, Sekhar AC, Upreti R. Physical impaction injury effects on bacterial cells during spread plating influenced by cell characteristics of the organisms. J Appl Microbiol 2014 Apr;116(4):911-922. 